University of Wollongong

Research Online

Faculty of Engineering and Information

Faculty of Engineering and Information

Sciences - Papers: Part B

Sciences

2017

Dynamic response of shear thickening fluid reinforced with $\mathrm{SiC}$ nanowires under high strain rates

Zhuhua Tan

Wuhan University of Technology

Jianhao Ge

Wuhan University of Technology

Hang Zhang

Lanzhou University of Technology

Pengcheng Zhai

Wuhan University of Technology

Weihua Li

University of Wollongong, weihuali@uow.edu.au

Follow this and additional works at: https://ro.uow.edu.au/eispapers1

Part of the Engineering Commons, and the Science and Technology Studies Commons

Research Online is the open access institutional repository for the University of Wollongong. For further information contact the UOW Library: research-pubs@uow.edu.au 


\title{
Dynamic response of shear thickening fluid reinforced with SiC nanowires under high strain rates
}

\author{
Abstract \\ In this letter, SiC nanowires were adopted to reinforce the nanoparticle-based shear thickening fluid (STF) \\ to improve its rheological properties. The reinforced STF showed a significant increase in viscosity. A \\ Split-Hopkinson pressure bar was implemented to evaluate the dynamic response of STF at strain rates in \\ the range of $3 \times 10^{3}-1.2 \times 10^{4} / \mathrm{s}$. For the pure STF, the flow stress reaches a saturation value with \\ increasing strain rates and shows almost no strain rate sensitivity, whereas the flow stress of the \\ reinforced STF increases with strain rates, and the strain rate sensitivity to flow stress is obvious owing to \\ the resistance of nanowires. The essence of this study is to reveal that there is a limiting value of the flow \\ stress of traditional nanoparticle-based STF at high strain rates due to the lubrication force among \\ particles. SiC nanowires can be used to break this limitation of the nanoparticle-based STF. \\ Disciplines \\ Engineering I Science and Technology Studies \\ Publication Details \\ Tan, Z., Ge, J., Zhang, H., Zhai, P. \& Li, W. (2017). Dynamic response of shear thickening fluid reinforced \\ with SiC nanowires under high strain rates. Applied Physics Letters, 111 (3), 031902-1-031902-4.
}

This journal article is available at Research Online: https://ro.uow.edu.au/eispapers $1 / 572$ 


\section{Dynamic response of shear thickening fluid reinforced with SiC nanowires under high strain rates}

Zhuhua Tan, Jianhao Ge, Hang Zhang, Pengcheng Zhai, and Weihua Li

Citation: Appl. Phys. Lett. 111, 031902 (2017); doi: 10.1063/1.4994066

View online: http://dx.doi.org/10.1063/1.4994066

View Table of Contents: http://aip.scitation.org/toc/apl/111/3

Published by the American Institute of Physics

\section{Articles you may be interested in}

Acoustical breakdown of materials by focusing of laser-generated Rayleigh surface waves

Applied Physics Letters 111, 031901 (2017); 10.1063/1.4993586

Enhanced radiative emission from monolayer $\mathrm{MoS}_{2}$ films using a single plasmonic dimer nanoantenna

Applied Physics Letters 111, 031101 (2017); 10.1063/1.4993427

Multi-frequency spin manipulation using rapidly tunable superconducting coplanar waveguide microresonators Applied Physics Letters 111, 032601 (2017); 10.1063/1.4993930

Acoustically driven particle delivery assisted by a graded grating plate

Applied Physics Letters 111, 031903 (2017); 10.1063/1.4991525

Low-cost fabrication of highly sensitive room temperature hydrogen sensor based on ordered mesoporous Codoped $\mathrm{TiO}_{2}$ structure

Applied Physics Letters 111, 032104 (2017); 10.1063/1.4994155

Semitransparent anisotropic and spin Hall magnetoresistance sensor enabled by spin-orbit torque biasing Applied Physics Letters 111, 032402 (2017); 10.1063/1.4993899

\section{A $|\mathrm{P}|$ Applied Physics Letters}

If your article has the

Save your money for your research. It's now FREE to publish with us no page, color or publication charges apply.

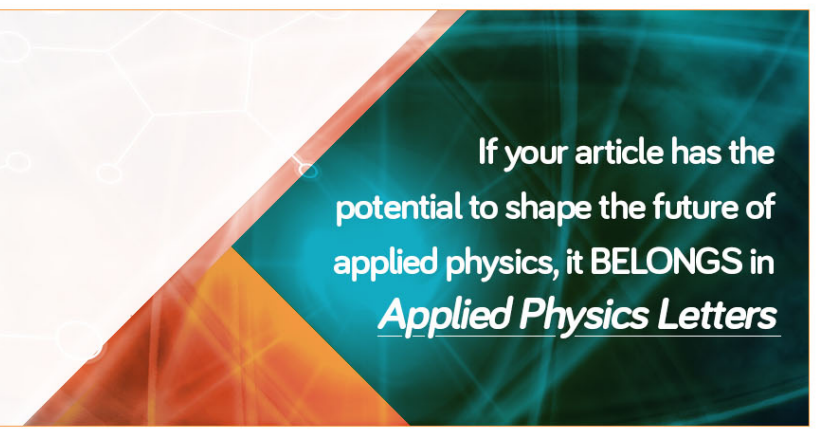




\title{
Dynamic response of shear thickening fluid reinforced with SiC nanowires under high strain rates
}

\author{
Zhuhua Tan, ${ }^{1, a)}$ Jianhao Ge, ${ }^{1}$ Hang Zhang, ${ }^{2}$ Pengcheng Zhai, ${ }^{1}$ and Weihua Li ${ }^{3, a)}$ \\ ${ }^{1}$ Hubei Key Laboratory of Theory and Application of Advanced Materials Mechanics, School of Science, \\ Wuhan University of Technology, Hubei 430070, People's Republic of China \\ ${ }^{2}$ School of Mechanical and Electronic Engineering, Lanzhou University of Technology, Lanzhou, 730050, \\ People's Republic of China \\ ${ }^{3}$ School of Mechanical, Materials and Mechatronic Engineering, University of Wollongong, Wollongong, \\ NSW 2522, Australia
}

(Received 24 April 2017; accepted 3 July 2017; published online 17 July 2017)

\begin{abstract}
In this letter, $\mathrm{SiC}$ nanowires were adopted to reinforce the nanoparticle-based shear thickening fluid (STF) to improve its rheological properties. The reinforced STF showed a significant increase in viscosity. A Split-Hopkinson pressure bar was implemented to evaluate the dynamic response of STF at strain rates in the range of $3 \times 10^{3}-1.2 \times 10^{4} / \mathrm{s}$. For the pure STF, the flow stress reaches a saturation value with increasing strain rates and shows almost no strain rate sensitivity, whereas the flow stress of the reinforced STF increases with strain rates, and the strain rate sensitivity to flow stress is obvious owing to the resistance of nanowires. The essence of this study is to reveal that there is a limiting value of the flow stress of traditional nanoparticle-based STF at high strain rates due to the lubrication force among particles. SiC nanowires can be used to break this limitation of the nanoparticle-based STF. Published by AIP Publishing. [http://dx.doi.org/10.1063/1.4994066]
\end{abstract}

The study on energy absorption and penetration resistance of materials/structures has attracted considerable interest in the field of armor systems. Many efforts have been done on materials such as metal foam, ${ }^{1}$ ceramic, ${ }^{2}$ and Kevlar fabrics. ${ }^{3}$ Recently, some works focused on the integration of shear thickening fluid (STF) in soft armor systems to improve ballistic performance. ${ }^{4-7}$ When the fabrics impregnated with STF are subjected to impact loading, the viscosity of STF in fabrics increases sharply at a certain shear rate. Part of the energy is dissipated through the increased viscosity of STF; ${ }^{8,9}$ further, the sliding of the fibers is prevented by the STF in the fabrics, which increases the loading capacity of the fabrics. Thus, viscosity is an essential property of STF required in various applications. ${ }^{10,11}$ However, the viscosity of STF relies on the lubrication force, which is influenced by the material, size, and volume fraction of the nanoparticles. $^{12-14}$ Currently, STF is mainly prepared from fumed silica or sub-micron particles, because of which it is difficult to improve the viscosity of STF based on nano-particles. ${ }^{15}$ To address this issue, we used $\mathrm{SiC}$ nanowires to reinforce the nanoparticle-based STF, which was expected to increase the viscosity by hindering the flow of nanoparticles.

In addition, we also tested the viscosity and dynamic flow stress of the reinforced STF to demonstrate the effectiveness of this method at high strain rates. STF undergoes high strain rate loading in the order of $10^{3}$ to $10^{5} / \mathrm{s}$ in applications involving energy absorption and vibration control, such as fabrics impregnated with STF against ballistic loading $^{16,17}$ and vibration control by squeeze flow of STF, ${ }^{18}$ whereas at present, the dynamic behavior of STF is mainly characterized by a rheometer at strain rates of the order of $10^{3} / \mathrm{s}{ }^{19,20}$ Moreover, some studies have been conducted on

\footnotetext{
${ }^{\text {a) }}$ Authors to whom correspondence should be addressed: zhtan@whut.edu.cn and weihuali@uow.edu.au
}

the numerical simulation of fabric or squeeze flow with $\mathrm{STF}^{21}$ where the dynamic material parameters at high strain rates are in urgent demands.

In this letter, $\mathrm{SiC}$ nanowires were used to reinforce pure silica nanoparticle-based STF, and then the dynamic response of the pure and reinforced STFs at high strain rates was investigated using the Split-Hopkinson pressure bar (SHPB). Pure STF consists of silica nanoparticles and polyethylene glycol, and the silica nanoparticles with a diameter approximately $400 \mathrm{~nm}$ were fabricated using the Stober method. Then, the specific amount of $\mathrm{SiC}$ nanowires [product of Nanjing XF Nano Company, as shown in Fig. 1(a)] was dispersed by the ultrasonic method and added into pure STF to improve the rheological properties. The total volume fraction of the solid phase in the STF was 56 vol. \%, as shown in Table I.

The viscosities of STFs with/without $\mathrm{SiC}$ nanowires were tested at shear rates in the range of $10^{-3}-100 / \mathrm{s}$ with a rheometer (Anton-Paar MCR301) at a temperature of $25^{\circ} \mathrm{C}$. As shown in Fig. 1(b), there are three phases in each curve: shear thinning, shear thickening, and again shear thinning. The viscosity of the reinforced STF is significantly higher compared to that of pure STF. At low shear rates, the viscosity values of the STF reinforced with $0.625 \mathrm{vol}$. \% and 1.25 vol. \% SiC nanowires are about $300 \mathrm{Pas}$ and $750 \mathrm{Pas}$, respectively, which are several times larger than those of pure STF. In the shear thickening phase, the maximum viscosities of STF with and without SiC nanowires are approximately $587 \mathrm{Pas}$ and $465 \mathrm{~Pa} \mathrm{~s}$, respectively, and the increase in amplitude is approximate to $26 \%$. Although the viscosity of the STF with $1.25 \mathrm{vol}$. \% SiC nanowires is the highest at the low strain rate, the viscosity in the shear thickening zone is approximately $300 \mathrm{~Pa} \mathrm{~s}$, which is almost the same as pure STF but less than the STF with 0.625 vol. \% SiC nanowires. We considered that the excessive quantity of nanowires 

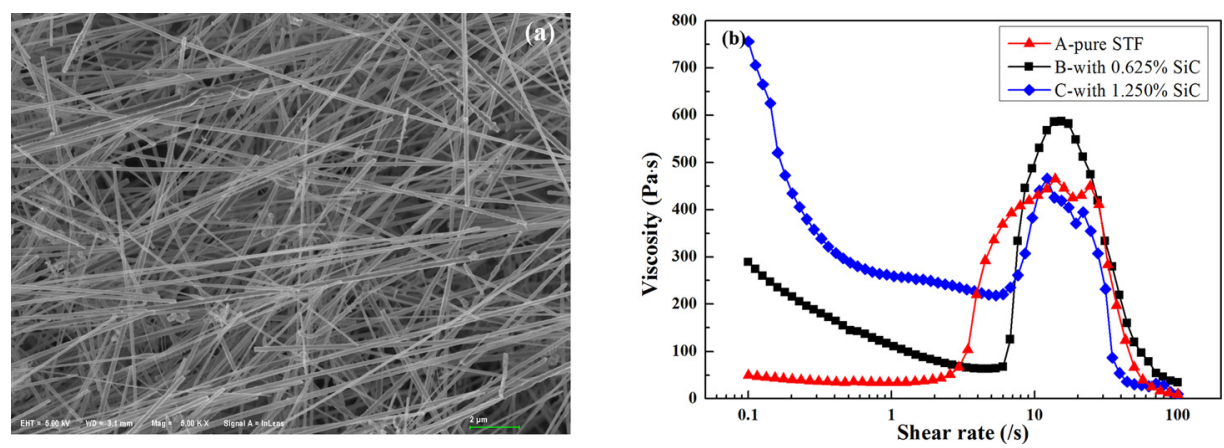

FIG. 1. SiC nanowires in STF and its viscosity. (a) SEM graphs of SiC nanowires; and (b) Curves of viscosityshear rates of samples A, B, and C.

TABLE I. Volume fraction of the solid phase in different STFs.

\begin{tabular}{lccc}
\hline \hline Sample No. & A & B & C \\
\hline Volume fraction of $\mathrm{SiC}$ nanowires (vol. \%) & 0 & 0.625 & 1.250 \\
Volume fraction of $\mathrm{SiO}_{2}($ vol. \%) & 56 & 55.375 & 54.750 \\
\hline \hline
\end{tabular}

presented the nanoparticles in the case of $1.25 \mathrm{vol} . \%$, which made the chain of nanoparticles unstable.

The SHPB is widely used to characterize dynamic mechanical properties of materials, which consists of a strike bar, an incident bar, and a transmission bar. ${ }^{7,22,23}$ During the test, the incident wave, reflection wave, and transmission wave are recorded using strain gauges mounted on the incident and transmission bars. The dynamic stress and strain can be calculated using the following formula:

$$
\sigma=\frac{A_{B}}{A_{S}} E \varepsilon_{T} \quad \varepsilon=\frac{2 c}{l} \int \varepsilon_{R} d t \quad \dot{\varepsilon}=\frac{2 c}{l} \varepsilon_{R},
$$

where $\sigma$ and $\varepsilon$ are the dynamic stress and strain, respectively; $c$ is the stress wave velocity in the pressure bar; $\varepsilon_{R}$ and $\varepsilon_{T}$ are the reflection and transmission stress waves, respectively; $A_{B}$ and $A_{s}$ and $l$ are the cross-sectional area of the bar and specimen and the length of the specimen, respectively. All the pressure bars are made of aluminum due to the low impedance of STF. The pressure bar is $40 \mathrm{~mm}$ in diameter, the incident and transmission bars are both $1800 \mathrm{~mm}$ in length (elastic modulus $E_{A L}=70 \mathrm{GPa}$, density $\rho=2.71 \mathrm{~g} / \mathrm{cm}^{3}$, and wave velocity $c=5218 \mathrm{~m} / \mathrm{s}$ ), and the strike bar is $300 \mathrm{~mm}$ long. The schematic graph of SHPB is shown in Fig. 2. The sampling rate is $5 \mathrm{MHz}$. The specimen is $1 \mathrm{~mm}$ in thickness and $40 \mathrm{~mm}$ in diameter. ${ }^{22-24}$

A typical stress wave obtained in the experiments is shown in Fig. 3(a). The dynamic stress-strain curves are shown in Figs. 3(b)-3(d). The strain rate has a significant influence on the flow stress in all the STF samples. The flow stress of pure STF increases as the strain rate increases, but such an increasing tendency decreases when the strain rate

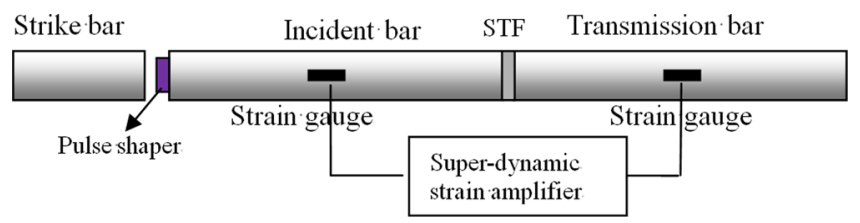

FIG. 2. Schematic graph of SHPB in the experiment. exceeds 5000/s. In addition, the flow stress of pure STF reaches a saturated value of approximately $48.03 \mathrm{MPa}$ at higher shear rates. However, the STFs reinforced with $\mathrm{SiC}$ nanowires do not show such tendency and saturated value, and the flow stress continues to increase with the strain rate. Moreover, the maximum flow stress of the STF reinforced with $\mathrm{SiC}$ nanowires is approximately $70 \mathrm{MPa}$, which shows that $\mathrm{SiC}$ nanowires have a significant influence on the flow stress at different strain rates, as shown in Fig. 3(d).

In the SHPB tests, STF samples were compressed between two pressure bars, which can be considered as squeeze flow, as shown in Fig. 3(e). According to the squeeze flow theory, ${ }^{15}$ both normal and shear stresses in the STF are induced by dynamic compression. In the case of pure STF, the lubrication force among the nanoparticles suffers the flow stress produced at low strain rates, namely, the flow stress can increase with the strain rate within the range of the lubrication force. Once the flow stress is larger than the lubrication force at a certain strain rate, STF would flow in the radial direction, which results in no obvious increment in flow stress at high strain rates, namely, the flow stress reaches a saturated value. In the case of reinforced STF, although the squeeze force acts on the STF, SiC nanowires prevent the flow of nanoparticles in the radial direction, which results in an increase in flow stress. Therefore, the greater the squeeze force, the larger the increase in flow stress, which corresponds to the strain rate sensitivity to flow stress in reinforced STF at high strain rates.

The peak stresses at different strain rates are shown in Fig. 4(a). At strain rates in the range of 3000/s-12 000/s, the peak stresses in pure STF are approximately $37.2 \mathrm{MPa}$, 45.6 $\mathrm{MPa}$, 47.9 $\mathrm{MPa}$, and $48.1 \mathrm{MPa}$, whereas those of the $\mathrm{STF}$ reinforced with $1.25 \mathrm{vol}$. \% $\mathrm{SiC}$ nanowires are 41.86 MPa, 52.86 MPa, 63.87 MPa, and 74.41 MPa. It is clear that the reinforced STFs show significant strain rate sensitivity compared to pure STF.

The energy absorption $E$ is defined as ${ }^{25}$

$$
E=E_{I-S}-E_{T-S}=\frac{c_{B} A_{B}}{E_{B}} \int_{0}^{T} \sigma_{I-S}^{2}(t) d t-\frac{c_{B} A_{B}}{E_{B}} \int_{0}^{T} \sigma_{T-S}^{2}(t) d t,
$$

where $E_{I-S}$ and $\sigma_{I-S}$ are the incident energy and incident pulse, respectively, at the front specimen-bar interface; $E_{T-S}$ and $\sigma_{T-S}$ are the transmission energy and transmission pulse, respectively, at the back specimen-bar interface. The energy absorption levels at different strain rates are shown in Fig. 4(b). It is evident that the reinforced STF has a higher 

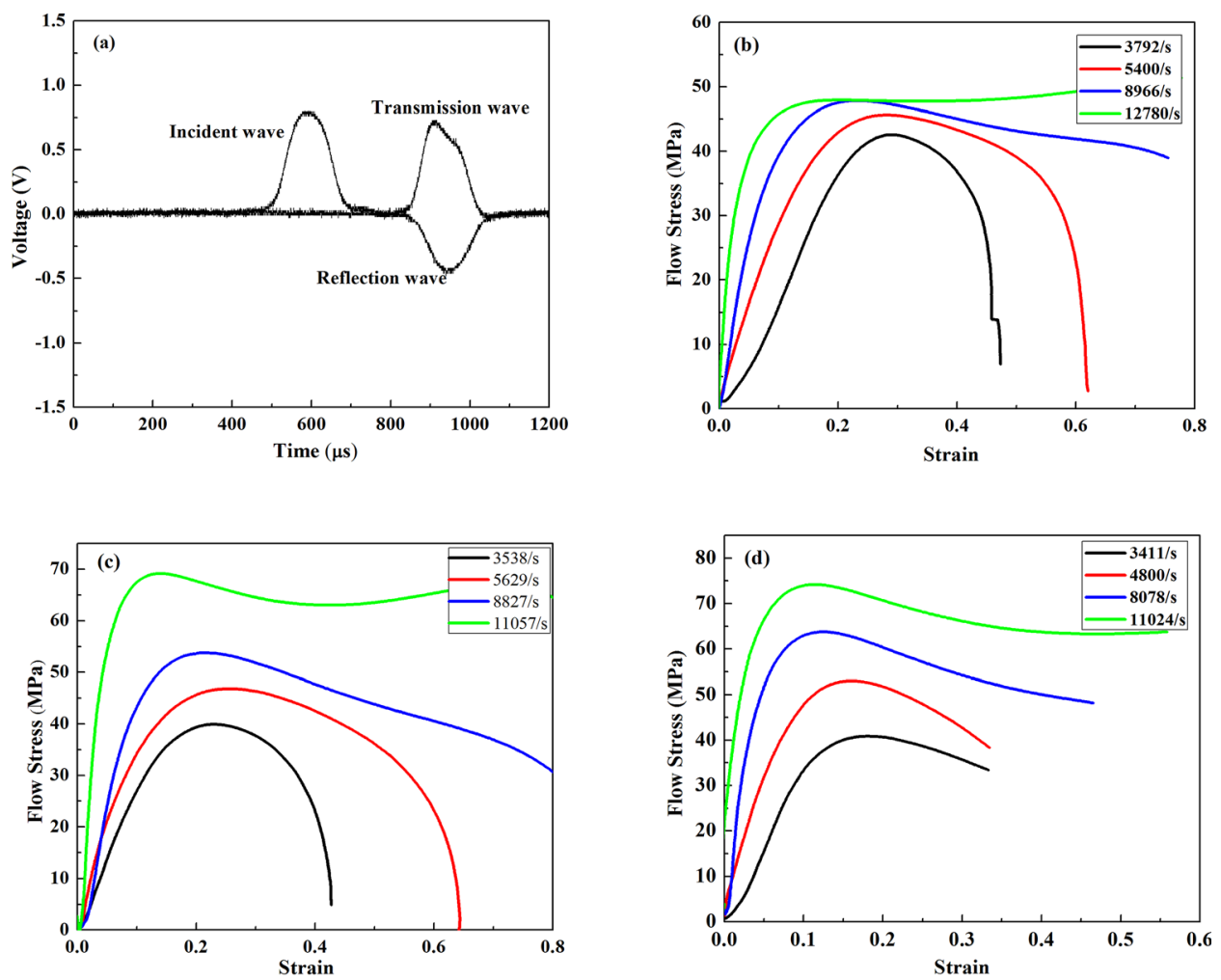

FIG. 3. SHPB experimental results at high strain rates. (a) Typical stress wave; (b)-(d) Dynamic stress-strain curves; (b) pure STF; (c) STF with 0.625 vol. \% SiC nanowires; (d) STF with 1.250 vol. \% SiC nanowires; and (e) schematic graph of the deformation mechanism of STF with/without SiC nanowires.

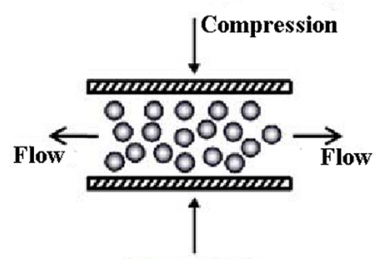

(Pure STF)

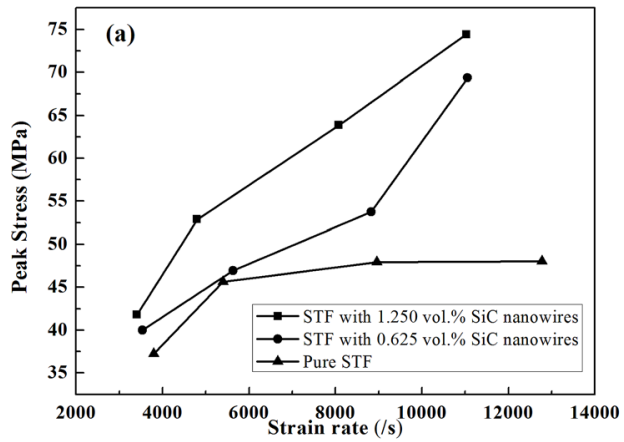

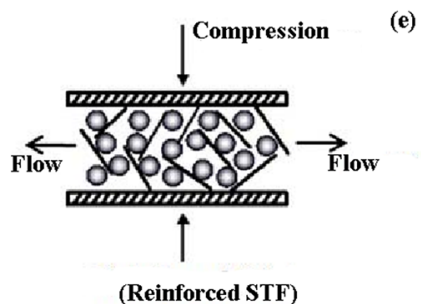

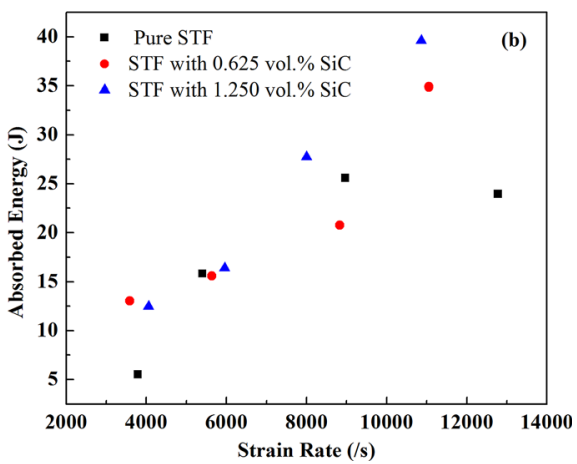

FIG. 4. Peak stress and energy absorption of STF. (a) Peak stress at different strain rates; and (b) Energy absorption at different strain rates. energy absorption capacity compared to the pure STF. The tendency is in agreement with the strain rate sensitivity of the STF.

In summary, $\mathrm{SiC}$ nanowires were used to reinforce the STF, which resulted in a significant increase in viscosity compared to pure STF. In the SHPB experiments, the flow stress of pure STF reached a saturated value, whereas reinforced STF showed a significant increase in flow stress with strain rate. We considered that $\mathrm{SiC}$ nanowires prevented the flow of silica nanoparticles in the reinforced STF, which increased the normal and shear stresses at high strain rates. It means that the reinforced STF can bear a larger dynamic loading than pure STF, and this improvement will be useful in future applications.

This work was supported by the National Natural Science Foundation of China (No. 11672214) and the Fundamental Research Funds for the Central Universities, Wuhan University of Technology (WUT: 2017IB008).

${ }^{1}$ D. Karagiozova, G. Langdon, and G. Nurick, Int. J. Solids Struct. 49(19), 2763 (2012).

${ }^{2}$ Z. Tan, X. Han, W. Zhang, and S. Luo, Int. J. Impact Eng. 37(12), 1162 (2010). 
${ }^{3}$ M. V. Hosur, U. K. Vaidya, C. Ulven, and S. Jeelani, Compos. Struct. 64, 455 (2004).

${ }^{4}$ V. B. C. Tan, T. E. Tay, and W. K. Teo, Int. J. Solids Struct. 42, 1561 (2005). ${ }^{5}$ J. L. Park, B. I. Yoon, J. G. Paik, and T. J. Kang, Text. Res. J. 82, 542 (2012).

${ }^{6}$ Y. Xu, X. Chen, Y. Wang, and Z. Yuan, Compos. Struct. 163, 465 (2017).

${ }^{7}$ S. Cao, Q. Chen, Y. Wang, S. Xuan, W. Jiang, and X. Gong, Composites, Part A 100, 161 (2017).

${ }^{8}$ I. R. Peters, S. Majumdar, and H. M. Jaeger, Nature 532, 214 (2016).

${ }^{9}$ W. Jiang, S. Xuan, and X. Gong, Appl. Phys. Lett. 106, 151902 (2015).

${ }^{10}$ S. S. Iyer, R. Vedad-Ghavami, H. Lee, M. Liger, H. P. Kavehpour, and R. N. Candler, Appl. Phys. Lett. 102, 251902 (2013).

${ }^{11}$ X. Q. Wu, F. C. Zhong, Q. Y. Yin, and C. G. Huang, Appl. Phys. Lett. 106, 071903 (2015).

${ }^{12}$ O. E. Petel and A. J. Higgins, J. Appl. Phys. 108, 114918 (2010).

${ }^{13}$ O. E. Petel, D. L. Frost, A. J. Higgins, and S. Ouellet, Phys. Rev. E 85, 021401 (2012).

${ }^{14}$ K. Chen, Y. Wang, S. Xuan, and X. Gong, J. Colloid Interface Sci. 497, 378 (2017).
${ }^{15}$ A. Laha and A. Majumdar, Appl. Clay Sci. 132-133, 468 (2016).

${ }^{16}$ O. E. Petel, S. Ouellet, J. Loiseau, B. J. Marr, D. L. Frost, and A. J. Higgins, Appl. Phys. Lett. 102, 064103 (2013).

${ }^{17}$ O. E. Petel and J. D. Hogan, Int. J. Impact Eng. 93, 39 (2016).

${ }^{18}$ C. Guo, X. Gong, S. Xuan, Q. Yan, and X. Ruan, Smart Mater. Struct. 22, 045020 (2013).

${ }^{19}$ R. L. Hoffman, Trans. Soc. Rheol. 16, 155 (1972).

${ }^{20}$ H. A. Barnes, J. Rheol. 33, 329 (1989).

${ }^{21}$ Z. Lu, L. Wu, B. Gu, and B. Sun, Composites, Part B 69, 191 (2015).

${ }^{22}$ A. S. Lim, S. L. Lopatnikov, and J. W. Gillespie, Jr., Polym. Test. 28, 891 (2009).

${ }^{23}$ A. S. Lim, S. L. Lopatnikov, N. J. Wagner, and J. W. Gillespie, Jr., Rheol. Acta 49, 879 (2010).

${ }^{24}$ N. Asija, H. Chouhan, S. A. Gebremeskel, and N. Bhatnagar, "High strain rate characterization of shear thickening fluids using Split Hopkinson Pressure Bar technique," Int. J. Impact Eng. (in press).

${ }^{25}$ W. F. Jiang, X. L. Gong, S. H. Xuan, W. Q. Jiang, F. Ye, X. F. Li, and T. X. Liu, Appl. Phys. Lett. 102, 101901 (2013). 